\title{
THE KRASSER COLLECTION IN THE FACULTY OF SCIENCES, CHARLES UNIVERSITY, PRAGUE - NEW INSIGHTS INTO THE MIDDLE JURASSIC FLORA OF SARDINIA
}

\author{
EVELYN KUSTATSCHER ${ }^{1, *}$, GIOVANNI G. SCANU ${ }^{2}$, JIŘí KVAČEK ${ }^{3}$, JOHANNA H. A. VAN KONIJNENBURG- \\ VAN CITTERT ${ }^{4}$
}

\begin{abstract}
'Museum of Nature South Tyrol, Bindergasse/via Bottai 1, 39100 Bozen/Bolzano and Department für Geo- und Umweltwissenschaften, Paläontologie und Geobiologie, Ludwig-Maximilians-Universität München und Bayerische Staatssammlung für Paläontologie und Geologie, RichardWagner-Straße 10, 80333 München, Germany; e-mail: evelyn.kustatscher@naturmuseum.it.

2 Dipartimento di Scienze Chimiche e Geologiche, Università degli Studi di Cagliari, via Trentino 51, 09127 Cagliari and AMAP CIRAD, Boulevard de la Lironde, 34398 Montpellier Cedex 5, France; e-mail: gioscanu@gmail.com.

${ }^{3}$ National Museum, Prague, Václavské nám. 68, 11579 Praha 1, the Czech Republic; e-mail: jiri_kvacek@nm.cz.

${ }^{4}$ Laboratory of Palaeobotany and Palynology, Heidelberglaan 2, 3584 CS Utrecht, The Netherlands and Naturalis Biodiversity Center, PO Box 9517, 2300 RA Leiden, The Netherlands; e-mail: jtvk@kgk.nl.

* corresponding author
\end{abstract}

Kustatscher, E., Scanu, G. G., Kvaček, J., Van Konijnenburg-van Cittert, J. H. A. (2016): The Krasser collection in the Faculty of Sciences, Charles University, Prague - new insights into the Middle Jurassic flora of Sardinia. - Fossil Imprint, 72(3-4): 140-154. Praha. ISSN 2533-4050 (print), ISSN 2533-4069 (online).

\begin{abstract}
Revision of part of the Middle Jurassic flora of Sardinia, the Krasser collection, stored in Prague (Lovisato B collection), containing 23 fossil taxa of horsetails, ferns, cycadophytes, ginkgophytes and conifers. The conifers are most diverse, followed by cycadophytes and ferns. The composition of this assemblage differs notably from the Lovisato collection stored in Cagliari, suggesting that it might derive from a different stratigraphic level and/or palaeoenvironment. The palaeodiversity of the Middle Jurassic flora of Sardinia increases to 46 fossil taxa with this revision. Cycadolepis sp. Nilssonia sp., Nilssonia sp. cf. N. orientalis, Pagiophyllum sp. and Agathoxylon sp. are described for the first time from the Middle Jurassic of Sardinia.
\end{abstract}

Key words: Fridolin Krasser, Italy, historic collections, horsetails, conifers, plant assemblages, biodiversity

Received: October 5, 2016 | Accepted: November 22, 2016 |Issued: December 30, 2016

\section{Introduction}

Jurassic floras are abundant in Europe, although not all are equally well understood. A recent overview documented 46 Early, Middle and Late Jurassic plant assemblages, with more than 770 taxa (for more details see Barbacka et al. 2014). The fossil sites from Yorkshire in the UK (e.g. Harris 1961, 1964, 1969, 1979, Harris et al. 1974, Van Konijnenburg-van Cittert and Morgans 1999, Cleal et al. 2001, Van Konijnenburg-van Cittert 2008), Franken in Germany (Weber 1968), Scania in Sweden (Pott and McLouglin 2011) and Anina in Romania (Popa and Meller 2009) are among the most famous European Jurassic floras. Italian Jurassic floras include the historical collections from Rotzo (de Zigno 1856-1868, 1873-1885, Grandori 1913a, b, Wesley 1956, 1958, 1966, 1974), Sardinia (Tornquist 1902, 1904a, b, Krasser 1912, 1913, 1920, Edwards 1929, Comaschi Caria 1959, Dieni et al. 1983, Salard-Cheboldaeff and VozeninSerra 1984, Scanu et al. 2012, 2015, 2016) and some smaller and less well studied plant assemblages (Sordelli 1896, Praturlon 1965, 1968, Cocozza and Praturlon 1966, Bonci and Vannucci 1986, Bravi 1995, Bravi et al. 2014). The floras of Rotzo, Como/Moltrasio and Monte Palombo are of Early Jurassic age, whereas those of Sardinia, Monti Lepini and Monte Fallano belong to the Middle Jurassic.

The largest assemblage of Sardinian plant fossils is housed in the Geological and Palaeontological Museum "D. Lovisato" of the Cagliari University. It comprises 24 taxa (19 genera) belonging to the horsetails, ferns (Phlebopteris, Hausmannia, Coniopteris, Todites, Cladophlebis), seed ferns (Sagenopteris, Ptilozamites), cycadophytes (Nilssonia, Pterophyllum, Cycadeospermum, Ptilophyllum, Williamsonia, Weltrichia), Czekanowskiales (Czekanowskia), conifers (Geinitzia, Brachyphyllum, Elatocladus) and plant remains without clear botanical affinity (Carpolithes, Taeniopteris; Scanu et al. 2012, 2015, 2016). Recently, a second collection (Miccolis collection) of Middle Jurassic plant remains from central Sardinia (Laconi and Nurallao) was discovered at the Natural History Museum of Venice, yielding several additional taxa (e.g. Marattia, Dicksonia, Eboracia). This increases the number to 29 taxa (23 genera), and reveals that the Middle Jurassic flora of Sardinia was more similar to the 
Middle Jurassic flora of Yorkshire than to the Early Jurassic flora of Rotzo (Italy, Scanu et al. 2016). Whether the difference between the two Italian floras (Sardinia and Rotzo) is due to their different depositional/environmental conditions, or reflects the palaeogeographic position of the floras during the Jurassic, remains equivocal (e.g. Bartiromo and Barone Lumaga 2009, Barbacka et al. 2014, Scanu et al. 2016). Moreover, it emphasizes that the Middle Jurassic flora of Sardinia is not yet fully understood, since the discovery of the small Miccolis collection in Venice added a significant number of new taxa to the Sardinian flora.

This study evaluates the composition of the second part of the Lovisato collection, stored in the Faculty of Sciences, Charles University, Prague, and adds new taxon records to the Jurassic flora of Sardinia. All historical collections have now been revised, providing complete available data on the composition of the Jurassic flora of Sardinia; Urbanisation of the area in the last century has impeded further collection of Jurassic plant remains from this area.

\section{Material and methods}

Domenico Lovisato (1842-1916), Professor of Mineralogy and Geology, and Director of the Royal Mineralogical and Geological Museum of the University of Cagliari collected the largest Middle Jurassic plant assemblage in Italy. This collection (about 1200 plant fossils), partly studied and described by Tornquist (1902, 1904a, b) and Krasser (1912, 1913), was severely damaged during the World War II. About 500 slabs, housed at the Geological and Palaeontological Museum of the Cagliari University, have been recently revised (Scanu et al. 2012, 2015). A selection of taxa (collected after 1913), was sent to Prague for taxonomical analyses to be carried out by Fridolin Krasser (Krasser 1920, Edwards 1929) were they are still stored.

The plant remains come from the Genna Selole Formation (Costamagna 2015), a siliciclastic to mixed siliciclastic-carbonate succession (Costamagna and Barca 2004). Palynomorphs and molluscs date the formation to the Bajocian - Bathonian (Amadesi et al. 1960, Del Rio 1976, 1984, Dieni et al. 1983, Dieni and Massari 1985). The base is represented by continental sediments (fluvial conglomerates) that grade into transitional and coastal sediments (distal alluvial fan to transitional lagoonal littoral; see also Scanu et al. 2012 and ref. therein). The top of the formation is characterised by tidally influenced lagoonal sediments (e.g. Amadesi et al. 1960, Dieni et al. 1983, Dieni and Massari 1985).

According to Krasser's numbering, the collection he studied contained at least 190 specimens denoted "Loc. B", thus sent to him after 1913 (Krasser 1920). Only 143 specimens (prefix "JSK") containing almost 200 plant fragments, were found in the collections of the Faculty of Sciences, Charles University, Prague. They are preserved as impressions and compressions in fine-grained sediments (siltstones and marls) typical of the Genna Selole Formation. Photographs were taken with a digital system camera (Panasonic Lumix DMC-LX5). The part of the Lovisato collection described by Krasser (1912) is also numbered, but kept separate (Loc. A of Krasser 1920).
Where possible, cuticles were prepared according to the standard procedures of the Schulze method $\left(\mathrm{KClO}_{3}\right.$ and $30 \%$ $\mathrm{HNO}_{3} /$ neutralization in $5 \% \mathrm{NH}_{4} \mathrm{OH}$ ) as described by Kerp (1990) and Batten (1999).

\section{Plant fossils in the Faculty of Sciences, Charles University, Prague}

\section{Sphenophyta}

Decorticated stem fragments and diaphragms of horsetails are common in the collection. Based on their dimensions (mostly the diameter of their axes), they can be segregated into two fossil taxa, Equisetites beanii (BUNBURY) SEWARD, 1894 and Equisetites columnaris (Brongniart) Phillips, 1875. Most fragments belong to E. columnaris (P1. 1, Figs 1-3) characterized by narrower axes, that are generally 40-60 mm wide (Lov. B 2.1-2.3, $4.1-2,5,7-10,12,18,22.1-2,23.1-3,25,28.1,32$, 51.1-3, 52-54, 54.1-2, 56, 59-60, 72). External preserved features are impressions of the leaf sheaths $(37 \times 10 \mathrm{~mm})$ and microphylls ( $2 \mathrm{~mm}$ wide, with pointed apices; Lov. B 3.1, 4.1-4.2, 5, 8). Equisetites beanii (P1. 1, Figs 4-5) was probably a larger plant, with axes reaching up to $100 \mathrm{~mm}$ in width (Lov. B 1, 3.1, 6, 11, 16-17, 21, 25). The fossil stem fragments are generally smooth, because leaf sheaths fall off easily. Equisetites beanii is a typical Middle Jurassic taxon resembling the Triassic equivalent Equisetites arenaceus (JAEGER) SCHENK, 1864, whereas E. columnaris has been described from both Lower and Middle Jurassic strata (Poland: Jarzynka and Pacyna 2015, England: Harris 1961).

Krasser (1920: 4) listed only one horsetail species (Equisetites columnaris), because he considered $E$. beanii to be the thicker stems belonging to E. columnaris. In this case, $E$. beanii would represent the older, defoliated stems of $E$. columnaris (Schweitzer et al. 1997: 135-141). If both taxa belong to the same biological species, the specific name $E$. columnaris would have priority. In the Jurassic flora of Yorkshire, both species are present, with E. columnaris being more common than $E$. beanii. Both species may occur in the same deposits, but, at some localities, only one species occurs. For example, only E. columnaris is known from Hasty Bank, while the cliffs around Scarborough have yielded only E. beanii.

One plant fossil (Lov. B 49.1-2; Pl. 1, Figs 10-11) represents a nodal fragment of Schizoneura SCHIMPER et MougeOT, 1844 with microphylls, which seem to arise from the same position on the axis and are basally connate. Krasser (1920: 9) indicated that this fragment might represent a badly preserved basal frond fragment of Dictyophyllum rugosum LindLey et HutTon, 1834, whereas the label designates it Equisetites beanii (“Asterophyllites Beblätterung”). Furthermore, Krasser (1920) mentioned that the same specimen contained a fragment of Sagenopteris goeppertiana DE ZIGNO, 1865 (on p. 8) and Nilssonia compta (PhILlips) BRONGNIART, 1828 (on p. 9), but none of those could be identified on the slab. 


\section{Pteridophyta}

The fern frond fragments belong to three genera: Phlebopteris Brongniart, 1836, Todites SEward, 1900 and Cladophlebis BRONGNIART, 1849, all preserved as small fragments. Phlebopteris is represented by eight specimens, three of which belong to Phlebopteris polypodioides Brongniart, 1836 (Lov. B 17, 25, 95) (P1. 1, Fig. 8). The fragments are up to $40 \mathrm{~mm}$ long and $30 \mathrm{~mm}$ wide, with a distinct midrib and reticulate veins in the outer half of the lamina; Lov. B 95 is a fertile fragment, with sori. One fertile fragment (Lov. B 37), bearing circular sori (0.5-1.2 mm diameter) arranged in two rows close to the midrib, could also belong to this species.

Phlebopteris dunkeri (SCHENK) SCHENK, 1975 (Pl. 1, Figs 6,7$)$ is represented by small fragments with distinct anastomosing venation and a fusain-like preservation (Lov. B $64,73.1-2,78)$. Other pinna fragments $(8 \times 4 \mathrm{~mm})$ arising from the frond fragments (up to $45 \times 20 \mathrm{~mm}$ ), are not attributable to any species (Lov. B 77.1-2). Krasser (1920: 8) assigned the Phlebopteris (Laccopteris PRESL, 1838 being a junior synonym of Phlebopteris Brongniart, 1828) specimens from the Lovisato B collection to Laccopteris woodwardii (LECKENBY) SEWARD, 1910 (Lov. B 87, lost); Harris (1961) questioned this attribution to $P$. woodwardii. Krasser (1920) also mentioned Laccopteris elegans PrESL in STERNBERG, 1838 (Lov. B 37) [which is a synonym of $P$. braunii (GOEPPERT) HARRIS, 1980)], L. polypodioides (BRONGNIART) SEWARD, 1910 (Lov. B 73) and "L. spectabilis STUR" (Lov. B 64), the latter being a nomen nudum. According to Krasser (1913: 31) L. dunkeri was restricted to the white sandstone and L. elegans (= Phlebopteris braunii) to the grey marls, but, in the Lovisato B collection, both taxa occur in grey marls. Unfortunately, neither P. braunii nor L. spectabilis can be confirmed for this collection.

Nine frond fragments belong to Cladophlebis or Todites. Two samples (Lov. B 16, 43) represent Todites williamsonii (Brongniart) SEward, 1900 emend. Harris 1961 (Pl. 1, Fig. 9). The frond fragments are up to $35 \mathrm{~mm}$ long and $30 \mathrm{~mm}$ wide. Pinnae fragments up to $20 \mathrm{~mm}$ long and $10-15 \mathrm{~mm}$ wide arise from the $2-2.5 \mathrm{~mm}$ wide rachis. The slightly falcate pinnules arise at an acute angle; they are 6-9 $\mathrm{mm}$ long and 2-2.5 mm wide. The others (Lov. B 6, 9, 16.4, 28.4, 53, 55, 57.1+2) are too badly preserved for any species determination, and are assigned to Cladophlebis sp. Krasser (1913: 31, 1920: 9) listed only Todites williamsonii for the Lovisato B collection.

\section{Pteridospermatophyta}

The collection yielded four badly preserved leaflets (Lov. B 17, 35.1, 55, 57.1-2) of Sagenopteris PrEsL in SternBERG, 1838 (Pl. 2, Fig. 2). The isolated leaflets are asymmetrically lanceolate, up to $80 \mathrm{~mm}$ long and $30 \mathrm{~mm}$ wide, with a characteristic net venation. Several of the specimens mentioned by Krasser (1920: 10) as Sagenopteris goeppertiana DE ZIGNO, 1885 are lost; the remaining samples are not sufficiently preserved for any species determination. The "sporocarps" identified by Krasser (1920: 8) represent the remains of some branchiopods formerly called Estheria (Pl. 3, Figs 4, 6). These aquatic (fresh-water and mainly salt-water) crustaceans occur sporadically in non-marine facies, and are abundant from the Devonian to present (Chen 2008).

\section{Ginkgophyta}

A few leaf fragments (Lov. B 66.1, 66.2, 67.2, 94) may belong to the ginkgophyte Eretmophyllum THOMAs, 1913 (P1. 2, Figs 5,7). The fragments are up to $30 \mathrm{~mm}$ long and $12 \mathrm{~mm}$ wide, with a rounded apex and distinct, parallel veins. Krasser (1920: 14) attributed them to the conifer Podozamites lanceolatus (LINDLEY et HUTTON) BRAUN, 1843. However, Edward's (1929) E. lovisatoi EDWARDS, 1929 from Sardinia (see below) is confirmed by cuticle analyses. Harris et al. (1974) compared it with Yorkshire species of Eretmophyllum, considering them different mainly in their cuticular anatomy.

\section{Cycadophyta}

The Cycadales (or Nilssoniales) are represented by leaf fragments of Nilssonia BronN, 1835 (Lov. B 16, 35.1, 37). The best-preserved specimen (Lov. B 35.1) is $120 \mathrm{~mm}$ long and $40 \mathrm{~mm}$ wide, with a $3 \mathrm{~mm}$ wide rachis and a $30 \mathrm{~mm}$ broad half lamina, with distinct parallel and undivided secondary veins. Krasser (1920) indicated Nilssonia compta (Phillips) Brongniart, 1828 for the assemblage, however, the material is not well enough preserved (lacking cuticle) to confirm this attribution. Moreover, both specimens seem to be entire-margined Nilssonia leaves, like those of N. orientalis HEER, 1878 (see Schweitzer et al. 2000) (P1. 2, Fig. 1).

Bennettitalean leaves are represented by rare and fragmentary leaves of Ptilophyllum MorRIS in GrANT, 1840 (Lov. B 8, 16.1-3, 16.5, 21, 25, 63.1, 65, 92.1). The fragments are up to $45 \mathrm{~mm}$ long and $50 \mathrm{~mm}$ wide. The lanceolate to slightly falcate segments are $10-25 \mathrm{~mm}$ long and 2-3 mm wide, with distinct parallel veins (Pl. 2, Figs 4, $6)$. The specimens are too fragmentary to determine whether they belong to the previously described species Ptilophyllum pecten (Phillips) SEWARD, 1900 (Krasser 1920), Ptilophyllum pectinoides (Phillips) Halle, 1913 or Ptilophyllum cariae ScANU et al. 2015 (Scanu et al. 2015). Reproductive organs are represented by fragments of Williamsonia CARruthers, 1870 emend. Harris 1969 (Lov. B 16.6, 44.1-2). Krasser (1920: 14) attributed them to Williamsonia acuminata (DE ZIGNO) KRASSER, 1912 but the material is too badly preserved for a specific determination. The collection also includes isolated linear-lanceolate scales of Cycadolepis SAPORTA, 1875 (Lov. B 44.3-4, xy1).

\section{Coniferophyta}

The conifers are among the most abundant groups in this plant assemblage. Geinitzia divaricata (BUNBURY) HARRIS, 1979 is one of the most common taxa (Pl. 3, Figs 1, 7). The shoot fragments (Lov. B 3.2, 5, 10, 12, 15, 17, 22.1-2, $28.1-4,30.1-2,35.1,37,55,81,92.1-3,109)$ are up to $40 \mathrm{~mm}$ long and $30 \mathrm{~mm}$ wide, with an axis of 1-2 mm width. Lateral shoots arise alternately at acute angles. Leaves are helically arranged, arch outward at a wide angle (70-90 $)$, and are attached by their entire base. They are falcate, with only one vein and a pointed or slightly rounded apex. They are $2-5 \mathrm{~mm}$ long and $0.5-2 \mathrm{~mm}$ wide. Krasser (1920: 18) attributed the specimens to Cheirolepis setosus (PHILLIPS) SEWARD, 1900, whereas Edwards (1929) assigned them to Cryptomerites divaricatus BUNBURY, 1851. 
Elatides williamsonii (LiNDLEY et HuTTON) NATHORST, 1897 is also common (Pl. 3, Figs 5, 9); it is characterized by elongated leaves of 5-8 $\mathrm{mm}$ length and 1-2 $\mathrm{mm}$ width, arranged in a loose helix (Lov. B 14.1-2, 17.1-2, 27.1-2, 28.2-3, 29, 51.2, 72.1-3, 78, 90, 94, 107.2, 122.1-3, 127.6, B, C1-5). Krasser (1920: 18) identified this species as Pagiophyllum williamsonii (BRONGNIART) SEWARD, 1900. Two specimens (Lov B 9, 127.9) with linear leaves are identified as Elatocladus sp. Several shoot fragments with broad, leathery leaves are assigned to Brachyphyllum sp. (Lov. B 56, 58), and other shoot fragments with very small $(<5 \mathrm{~mm}$ long, $1 \mathrm{~mm}$ wide) leaves to Pagiophyllum sp. (Lov. B 75.1-2, 99, 124, 126, 127.1-2, 127.5, 127.7-9) (P1. 3, Fig. 8).

The wood fragment (Lov. B 128), labelled as Dadoxylon sp. might belong to Agathoxylon Greguss, 1952. Krasser (1920: 20) established Araucarites sardinicus (KRASSER) KRASSER, 1920 (formerly Cycadeospermum sardinicum) for sub-triangular ovuliferous scales (Lov. B 50, 50.1-2, 55, 56, 57.1-2), each with a large single seed (Pl. 3, Figs 2-3). The scales are 20-25 $\mathrm{mm}$ long and 20-22 mm wide, with a central depression or seed of 15-17 × 10-12 mm. The ovuliferous cones (Lov. B 127.8, B) are too fragmentary for determination. Fragments resembling pine-like leaves, with a transversely wrinkled lamina (Lov. B 89, 91) were ascribed by Krasser (1920: 19) to Pityophyllum nordenskioldii (HEER) SEWARD, 1919; unfortunately, no cuticle is preserved to confirm this attribution.

\section{Unknown botanical affinity}

Several plant remains of unknown botanical affinity are also preserved in the plant assemblage, such as seeds of Carpolithes sp. 1 sensu Scanu et al. (2015; Lov. B 97.2) and Carpolithes sp. 2 sensu Scanu et al. (2015; Lov. B 66, 77.1-2) and root fragments (Lov. B 129.1-6). Several rock samples host plant fragments too badly preserved for determination. The collection also includes a single small impression of a narrow stem with sub-rhombic leaf scars, named Sardoa robitschekii KRASSER, 1920 (Lov. B 46; P1. 2, Fig. 3). The leaf traces resemble Bucklandia PrESL, 1825 as figured by Harris (1969: pl. 6, figs 1-5, 8), an attribution to the bennettitales cannot be excluded. However, the leaf traces are too badly preserved for confident identification.

\section{Considerations on plant fossils \\ from the Lovisato collection of Cagliari}

In the light of additional and better-preserved plant fossils from the Jurassic of Sardinia, determinations of some plant fossils of the Lovisato collection housed in Cagliari (Scanu et al. 2015) need to be revised. This includes the frond fragment of Hausmannia DUNKer, 1846 that closely resembles Hausmannia crenata (NATHORST) MoELler, 1902, a well-known species from the Late Triassic to Middle Jurassic of Sweden, Denmark (Bornholm), Poland, Russia, Iran and Japan (Schweitzer et al. 2009). The specimen described as Coniopteris simplex (LindLEy et HuTTON) HARRIS, 1961, might very well be a small fertile fragment of Coniopteris hymenophylloides (BRONGNIART) SEWARD, 1900. Moreover, some of the specimens attributed to Todites williamsonii (Scanu et al. 2015: pl. 2, fig. 1) may represent badly preserved specimens of Cladophlebis denticulata
(Brongniart) Fontaine, 1889. An unresolved problem is the attribution of Ptilozamites sp. cf. Ptilozamites blasii. Ptilozamites has never been described from the Middle Jurassic, but Ctenozamites, a morphologically similar genus, is well known from that interval. A recent discussion suggests that Ptilozamites and Ctenozamites belong to the same natural genus, based on their resemblance in pinna morphology and cuticle pattern (Popa and McElwain 2009). However, incontrovertible evidence for the synonymy of these genera is missing; thus, we retain them as distinct, but considering the fragmentary nature of the Sardinian remains, we prefer to assign them to Ctenozamites. Moreover, the specimens attributed to Pterophyllum thomasii HARRIS, 1969 (Scanu et al. 2015) probably represent badly preserved fragments of Ptilophyllum hirsutum THOMAS et BANCROFT, 1949. Some of the segments might have been attached to the upper side of the rachis, whereas in Pterophyllum they are attached laterally.

\section{Overview of the plant groups in the Middle Jurassic flora of Sardinia}

Considering all three collections (Lovisato A collection in Cagliari, Lovisato B collection in Prague and Miccolis collection in Venice), the Jurassic flora of Sardinia is represented by more than 660 rock slabs with vegetative shoots and leaves, reproductive organs and dispersed seeds. Plant fossils are preserved as impressions and compressions, in some cases yielding cuticles and in situ spores. At least 46 taxa have been identified belonging to horsetails, ferns, seed ferns, cycadophytes, Czekanowskiales and conifers. Plant remains belonging to lycophytes are absent from the Middle Jurassic flora of Sardinia, but they are represented by dispersed spores (Del Rio 1976, 1984).

Horsetails and ferns are generally poorly and fragmentarily preserved. Horsetail remains in the Cagliari and Venice collections are impressions of stem fragments, with distinct vascular bundles and strobili fragments (Scanu et al. 2015, 2016). Identifiable stem fragments, attributed to Equisetites columnaris and Equisetites beanii (which might belong to the same biological species) are preserved only in Prague. Calamites lehmanianus GOEPPERT, 1836, indicated by Meneghini (1857), cannot be confirmed for this flora.

Ferns are common and represented by several families. Dicksoniaceae is represented by frond fragments of Coniopteris sp. cf. C. hymenophylloides (BRONGNIART) SEWARD, 1900 emend. Harris 1961, Dicksonia kendallii HARRIS, 1961 and Eboracia sp. cf. Eboracia lobifolia (PHILlips) THOMAs, 1911 emend. Harris 1961 (Scanu et al. 2015, 2016). The number of Matoniaceae species described from the Middle Jurassic of Sardinia rises to four (Phlebopteris polypodioides, P. dunkeri, P. muensteri (SCHENK) HIRMER et HöRHAMMER, 1936 and $P$. braunii (GÖPPERT) HARRIS, 1980). Marattia intermedia (MÜNSTER) KILPPER, 1964 is the only representative of Marattiaceae; Hausmannia crenata (NATHORST) MOELLER, 1902 is the only member of Dipteridaceae. Osmundaceae is represented by Todites williamsonii (BRONGNIART) SEWARD, 1900 emend. Harris 1961 and Cladophlebis sp. cf. C. denticulata (BRONGNIART) FONTAINE, 1889 and small fragments attributed to Cladophlebis sp. The fern frond taxa Coniopteris (= Kylikipteris) arguta (LindLEY et HuTtON) SEwARD, 1900, 
Table 1. Overview of all Jurassic plant taxa identified from Sardinia by various researchers. Names are grouped in their major plant groups, but listed alphabetically within each group. Synonymous names are grouped together for better readability (abbreviations: cf. - uncertain determination, n.v. - non vidimus, Ed. - detemination based on figure by Edwards 1929).

\begin{tabular}{|c|c|c|c|c|c|c|c|c|c|c|c|c|c|}
\hline TAXA & 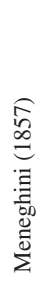 & 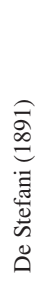 & 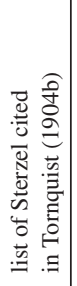 & 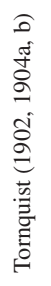 & 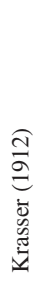 & 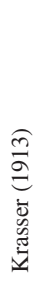 & 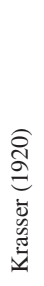 & 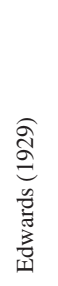 & 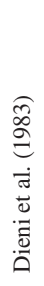 & 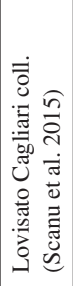 & 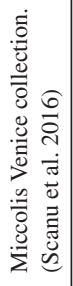 & 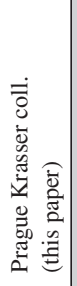 & 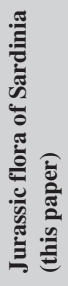 \\
\hline Sphenophytes & & & & & & & & & & $\mathrm{x}$ & $\mathrm{x}$ & & \\
\hline Calamites lehmanianus GOEPPERT, 1836 & $\mathrm{x}$ & & & & & & & & & & & & \\
\hline Calamites sp. & & & $\mathrm{x}$ & & & & & & & & & & \\
\hline Equisetites beanii (BUNBURY) SEWARD, 1851 & & & & & & & & & & & & $\mathrm{x}$ & $\mathbf{x}$ \\
\hline Equisetites columnaris BRONGNIART, 1828 & & & & & & & $\mathrm{x}$ & $\mathrm{x}$ & & & & $\mathrm{x}$ & $\mathbf{x}$ \\
\hline Equisetum arenaceum (JAEGER) SCHENK, 1864 & & cf. & $\mathrm{x}$ & & & & & & & & & & \\
\hline Equisetum mougeotii BRONGNIART, 1828 & & & $\mathrm{x}$ & & & & & & & & & & \\
\hline Schizoneura sp. & & & & & & & & & & & & cf. & $\mathbf{x}$ \\
\hline \multicolumn{14}{|l|}{ Pteridophytes } \\
\hline Caulopteris sp. & & & $\mathrm{x}$ & & & & & & & & & & \\
\hline Cladophlebis denticulata (BRONGNIART) FONTAINE, 1889 & & & & & & $\mathrm{x}$ & $\mathrm{x}$ & n.v. & & & & & cf. \\
\hline Cladophlebis sp. & & & & & & & & & & $\mathrm{x}$ & $\mathrm{x}$ & $\mathrm{x}$ & $\mathbf{x}$ \\
\hline Coniopteris (= Kylikipteris) arguta (LINDLEY et HUTTON) SEWARD, 1900 & & & & cf. & & & cf. & & & & & & \\
\hline Coniopteris hymenophylloides (BRONGNIART) SEWARD emend. Harris 1961 & & & & & & $\mathrm{x}$ & $\mathrm{x}$ & n.v. & & cf. & cf. & & ef. \\
\hline Coniopteris simplex (LINDLEY et HUTTON) HARRIS, 1961 & & & & & & & & & & $\mathrm{x}$ & & & \\
\hline Dicksonia kendallii HARRIS, 1961 & & & & & & & & & & & $\mathrm{x}$ & & $\mathbf{x}$ \\
\hline Dictyophyllum rugosum LiNDLEY et HuTTON, 1831 & & & & & & & $\mathrm{x}$ & $\mathrm{x}$ & & & & & \\
\hline Eboracia lobifolia (PHILLIPS) THOMAS, 1911 emend. Harris 1961 & & & & & & & & & & & cf. & & cf. \\
\hline Hausmannia crenata (NATHORST) MOELLER, 1902 & & & & & & & & & & sp. & & & $\mathbf{x}$ \\
\hline Klukia exilis (PHILLIPS) RACIBORSKI, 1890 & & & & & & $\mathrm{x}$ & $\mathrm{x}$ & n.v. & & & & & \\
\hline "Laccopteris spectabilis STUR" nom. nudum & & & & & & & $\mathrm{x}$ & & & & & & \\
\hline Marattia intermedia (MÜNSTER) KILPPER, 1964 & & & & & & & & & & & $\mathrm{x}$ & & $\mathbf{x}$ \\
\hline Phlebopteris (= Laccopteris) dunkeri (SCHENK) SCHENK, 1975 & & & & & & $\mathrm{x}$ & $\mathrm{x}$ & & & & & $\mathrm{x}$ & $\mathbf{x}$ \\
\hline Phlebopteris (= Laccopteris) polypoidioides BRONGNIART, 1836 & & & & & & & & $\mathrm{x}$ & & $\mathrm{x}$ & & $\mathrm{x}$ & $\mathbf{x}$ \\
\hline Phlebopteris (= Laccopteris) woodwardii LECKENBY, 1864 & & & & & & & $\mathrm{x}$ & $\mathrm{x}$ & & & & & \\
\hline $\begin{array}{l}\text { Phlebopteris braunii (GOEPPERT) HARRIS, } 1980 \text { = Laccopteris elegans } \\
\text { PRESL in STERNBERG, } 1838\end{array}$ & & & & & & $\mathrm{x}$ & $\mathrm{x}$ & & & & $\mathrm{x}$ & & $\mathbf{x}$ \\
\hline Phlebopteris muensteri (SCHENK) HIRMER et HÖRHAMMER, 1936 & & & & & & & & & & & $\mathrm{x}$ & & $\mathbf{x}$ \\
\hline Todites williamsoni (BRONGNIART) SEWARD emend. Harris 1961 & & & & & & $\mathrm{x}$ & $\mathrm{x}$ & n.v. & & cf. & & $\mathrm{x}$ & $\mathbf{x}$ \\
\hline \multicolumn{14}{|l|}{ Seed ferns } \\
\hline Ctenozamites sp. (= Ptilozamites blasii (BRAUNS) NATHORST, 1879) & & & & & & & & & & cf. & & & $\mathbf{x}$ \\
\hline $\begin{array}{l}\text { Caytonia } \text { sp. = Gristhorpia nathorstii THOMAS, } 1925=\text { Laconiella sardinica } \\
\text { KRASSER, } 1920\end{array}$ & & & & & & & $\mathrm{x}$ & $\mathrm{x}$ & & & & & Ed. \\
\hline Sagenopteris goeppertiana DE ZIGNO, 1885 & & & & & & $\mathrm{x}$ & $\mathrm{x}$ & $\mathrm{x}$ & & & & & \\
\hline Sagenopteris phillipsii (BRONGNIART) PRESL in STERNBERG, 1838 & & & & & & $\mathrm{x}$ & $\mathrm{x}$ & & & $\mathrm{x}$ & & & $\mathbf{x}$ \\
\hline Sagenopteris rhoifolia PRESL, 1838 var. elongata BRAUN, 1843 & & & $\mathrm{x}$ & & & & & & & & & & \\
\hline Sagenopteris sp. & & & $\mathrm{x}$ & & & & & & & & & $\mathrm{x}$ & \\
\hline \multicolumn{14}{|l|}{ Ginkgophytes } \\
\hline Baiera phillipsii NATHORST, 1880 & & & & & & $\mathrm{x}$ & $\mathrm{x}$ & n.v. & & & & & \\
\hline $\begin{array}{l}\text { Eretmophyllum lovisatoi EDWARDS, } 1929=\text { Podozamites lanceolatus } \\
\text { (LINDLEY et HUTTON) BRAUN, } 1843\end{array}$ & & & & & & & $\mathrm{x}$ & $\mathrm{x}$ & & & & $\mathrm{x}$ & $\mathbf{x}$ \\
\hline \multicolumn{14}{|l|}{ Czekanowskiales } \\
\hline $\begin{array}{l}\text { Czekanowskia furcula HARRIS et MILLER in HARRIS et al., } 1974= \\
\text { Czekanowskia murrayana (LINDLEY et HUTTON) SEWARD, } 1900\end{array}$ & & & & & & $\mathrm{x}$ & $\mathrm{x}$ & n.v. & & cf. & & & ef. \\
\hline \multicolumn{14}{|l|}{ Cycadophytes } \\
\hline Cycadeospermum lovisatoi KRASSER, 1912 & & & & & $\mathrm{x}$ & $\mathrm{x}$ & $\mathrm{x}$ & $\mathrm{x}$ & & $\mathrm{x}$ & & & $\mathbf{x}$ \\
\hline Cycadeospermum persicum KRASSER, 1912 & & & & & $\mathrm{x}$ & $\mathrm{x}$ & $\mathrm{x}$ & $\mathrm{x}$ & & & & & \\
\hline Cycadolepis sp. & & & & & & & & & & & & $\mathrm{x}$ & $\mathbf{x}$ \\
\hline Nilssonia compta BRONGNIART, 1828 & & & & & & $\mathrm{x}$ & $\mathrm{x}$ & $\mathrm{x}$ & & & & & \\
\hline Nilssonia orientalis HEER, 1878 & & & & & & & & & $\mathrm{x}$ & & & cf. & cf. \\
\hline Nilssonia sp. & & & & & & & & & & $\mathrm{x}$ & & & $\mathbf{x}$ \\
\hline Otozamites anglica (SEWARD) HARRIS, 1949 = Nageiopsis anglica SEWARD, 1900 & & & & & & $\mathrm{x}$ & $\mathrm{x}$ & n.v. & & & & & \\
\hline Otozamites beanii (LINDLEY et HUTTON) BRONGNIART, 1849 & & & & $\mathrm{x}$ & & & $\mathrm{x}$ & & & & & & \\
\hline Otozamites veronensis DE ZIGNO, 1881 = Otozamites lovisatoi KRASSER, 1913 & & & & & & $\mathrm{x}$ & $\mathrm{x}$ & $\mathrm{x}$ & & & & & Ed. \\
\hline Pterophyllum braunianum GOEPPERT, 1844 & & & cf. & & & & & & & & & & \\
\hline Pterophyllum jaegeri BRONGNIART, 1828 & & & cf. & & & & & & & & & & \\
\hline Pterophyllum thomasii HARRIS, 1969 & & & & & & & & & & cf. & & & \\
\hline Ptilophyllum (= Williamsonia/Pterophyllum) pecten (PHILLIPS) MORRIS, 1841 & & & & $\mathrm{x}$ & & $\mathrm{x}$ & $\mathrm{x}$ & $\mathrm{x}$ & & & & & \\
\hline Ptilophyllum cariae SCANU et al., 2015 & & & & & & & & & & $\mathrm{x}$ & & & $\mathbf{x}$ \\
\hline Ptilophyllum hirsutum THOMAS et BANCROFT, 1913 & & & & & & & & & $\mathrm{x}$ & & & & cf. \\
\hline Ptilophyllum pectinoides (PHILliPs) Halle, 1913 & & & & & & & & & & $\mathrm{x}$ & & & $\mathbf{x}$ \\
\hline
\end{tabular}


Table 1. - continued.

\begin{tabular}{|c|c|c|c|c|c|c|c|c|c|c|c|c|c|}
\hline TAXA & 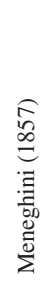 & 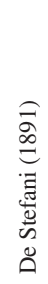 & 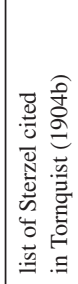 & 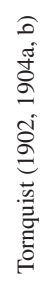 & 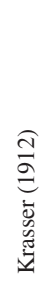 & 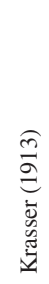 & 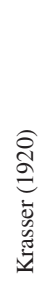 & 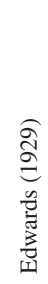 & 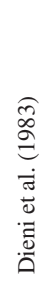 & 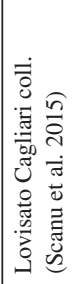 & 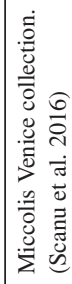 & 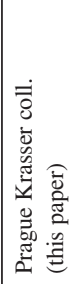 & 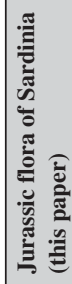 \\
\hline Ptilophyllum sp. & & & & & & & & & & & & $\mathrm{x}$ & $\mathbf{x}$ \\
\hline Taeniopteris vittata BRONGNIART, 1831 & & & & & & cf. & $\mathrm{x}$ & n.v. & & & & & \\
\hline Weltrichia (= Williamsonia) whitbiensis (NATHORST) HARRIS, 1969 & & & & & $\mathrm{x}$ & $\mathrm{x}$ & & & & & cf & & cf. \\
\hline Weltrichia sp. & & & & & & & & & & $\mathrm{x}$ & & & $\mathbf{x}$ \\
\hline Williamsonia acuminata (DE ZIGNO) KRASSER, 1920 & & & & & & $\mathrm{x}$ & $\mathrm{x}$ & & & & & & \\
\hline Williamsonia hildae HARRIS, 1969 & & & & & & & & & & $\mathrm{x}$ & & & $\mathbf{x}$ \\
\hline Williamsonia leckenbyi NATHORST, 1911 & & & & & $\mathrm{x}$ & $\mathrm{x}$ & $\mathrm{x}$ & $\mathrm{x}$ & & & & & \\
\hline Williamsonia sewardii KRASSER, 1913 & & & & & & & $\mathrm{x}$ & $\mathrm{x}$ & & & & & \\
\hline Williamsonia sp. & & & & & & & & & & & & $\mathrm{x}$ & \\
\hline Zamites sp. & & & & & & $\mathrm{x}$ & $\mathrm{x}$ & $\mathrm{x}$ & & & & & \\
\hline \multicolumn{14}{|l|}{ Coniferophytes } \\
\hline Agathoxylon sp. = Dadoxylon sp. & & & & & & & & $\mathrm{x}$ & & & & $\mathrm{x}$ & $\mathbf{x}$ \\
\hline Araucarites (= Cycadeospermum) sardinicus (KRASSER) KRASSER, 1920 & & & & & $\mathrm{x}$ & $\mathrm{x}$ & $\mathrm{x}$ & $\mathrm{x}$ & & & & $\mathrm{x}$ & $\mathbf{x}$ \\
\hline $\begin{array}{l}\text { Brachyphyllum (= Thuites) expansum (PRESL in STERNBERG) SEWARD, } 1919 \\
\text { emend. Kendall } 1949\end{array}$ & & & & & & $\mathrm{x}$ & $\mathrm{x}$ & n.v. & & $\mathrm{x}$ & & & $\mathbf{x}$ \\
\hline Brachyphyllum mamillare BRONGNIART, 1828 & & & & & & $\mathrm{x}$ & $\mathrm{x}$ & $\mathrm{x}$ & & & & & \\
\hline Brachyphyllum majus BRONGNIART, 1828 & $\mathrm{x}$ & & & & & & & & & & & & \\
\hline Brachyphyllum sp. & & & & & & & & & & $\mathrm{x}$ & & $\mathrm{x}$ & $\mathbf{x}$ \\
\hline Elatides (= Pagiophyllum) williamsonii (BRONGNIART) HARRIS, 1943 & & & & & & & $\mathrm{x}$ & $\mathrm{x}$ & & & & $\mathrm{x}$ & $\mathbf{x}$ \\
\hline Elatocladus sp. & & & & & & & & & & $\mathrm{x}$ & & $\mathrm{x}$ & $\mathbf{x}$ \\
\hline $\begin{array}{l}\text { Geinitzia }(=\text { Cryptomerites) divaricata (BUNBURY) HARRIS, } 1979= \\
\text { Cheirolepis setosus } \text { (PHILLIPS) SEWARD, } 1900\end{array}$ & & & & & & & $\mathrm{x}$ & $\mathrm{x}$ & & $\mathrm{x}$ & $\mathrm{x}$ & $\mathrm{x}$ & $\mathbf{x}$ \\
\hline Pagiophyllum sp. & & & & & & & & & & & & $\mathrm{x}$ & $\mathbf{x}$ \\
\hline Pityophyllum nordenskioldii (HEER) SEWARD, 1919 & & & & & & & $\mathrm{x}$ & & & & & & \\
\hline Albertia latifolia SCHIMPER, 1837 or Albertia braunii SCHIMPER, 1837 & & & $\mathrm{x}$ & & & & & & & & & & \\
\hline Voltzia sp. & & $\mathrm{x}$ & $\mathrm{x}$ & & & & & & & & & & \\
\hline $\begin{array}{l}\text { Yuccites dubius SCHIMPER et MOUGEOT, } 1844 \text { or Yuccites vogesiacus } \\
\text { SCHIMPER et MOUGEOT, } 1844\end{array}$ & & & $\mathrm{x}$ & & & & & & & & & & \\
\hline \multicolumn{14}{|l|}{ indet. } \\
\hline Carpolithes sp. 1 & & & & & & & & & & $\mathrm{x}$ & & $\mathrm{x}$ & $\mathbf{x}$ \\
\hline Carpolithes sp. 2 & & & & & & & & & & $\mathrm{x}$ & $\mathrm{x}$ & $\mathrm{x}$ & $\mathbf{x}$ \\
\hline Carpolithes sp. & & & & & & & $\mathrm{x}$ & & & & & & \\
\hline Sardoa robitschekii KRASSER, 1920 & & & & & & $\mathrm{x}$ & $\mathrm{x}$ & $\mathrm{x}$ & & & & $\mathrm{x}$ & $\mathbf{x}$ \\
\hline Taeniopteris sp. & & & & & & $\mathrm{x}$ & & & & $\mathrm{x}$ & & & $\mathrm{x}$ \\
\hline
\end{tabular}

Dictyophyllum rugosum Lindey et HutTon, 1831, Klukia exilis (PHILLIPS) RACIBORSKI, 1890 and "Laccopteris spectabilis STUR" (nom. nudum) were not confirmed for this flora (Tab. 1).

Gymnosperms are more abundant than the remains of spore-producing plants. Ctenozamites sp. and Sagenopteris phillipsii PRESL in STERnBERG, 1838 (rather than Sagenopteris goeppertiana DE ZIGNO, 1885) are the only seed ferns in the collections of Middle Jurassic plants from Sardinia (Scanu et al. 2015). Unfortunately, Laconiella sardinica KRASSER, 1920, moved by Edwards (1929) to Gristhorpia nathorstii THOMAS, 1925, could not be found in the collection. The photographs provided by Edwards (1929: pl. 4, figs 1-2) show a Caytonia-type of reproductive organ, which would fit perfectly with the Sagenopteris leaves found in the collection (Tab. 1). The specimens, attributed by Krasser (1920: 10) to Podozamites lanceolatus (LINDLEY et HUTTON) BRAUN, 1843, probably belong to the only putative ginkgophyte Eretmophyllum lovisatoi EDWARDS, 1929, whereas Baiera phillipsii NATHORST, 1880 could not be confirmed. Czekanowkiales is represented by Czekanowskia sp. cf. Czekanowskia furcula HARris et Miller in HARris et al., 1974, which might correspond to Czekanowskia murrayana (LINDLEY et HUTTON) SEWARD, 1900 as listed by Krasser $(1913,1920)$ and Edwards (1929).

The cycadophytes are the most important group, both in species diversity and relative abundance (Scanu et al. 2015). To the Cycadales belong dispersed leaf segments of Nilssonia sp., probably Nilssonia orientalis HEER, 1878 and the seed type Cycadeospermum lovisatoi KRASSER, 1912, which could belong to the former (Gothan 1914, Edwards 1929). The bennettitalean leaves are much more diverse, with leaf fragments of Ptilophyllum pectinoides (PHILliPs) Halle, 1913, Ptilophyllum cariae SCANu et al. 2015 (Scanu et al. 2015), and perhaps Ptilophyllum hirsutum Thomas et BANCROFT ex HARris, 1949 (Dieni et al. 1983, SalardCheboldaeff and Vozenin-Serra 1984). The reproductive organs Williamsonia hildae HARRIS, 1969 (female) and Weltrichia sp. cf. Weltrichia whitbiensis (NATHORST) HARRIS, 1969 (male) together with the leaves Ptilophyllum pectinoides, belong to the same natural species (Harris 1969, Scanu et al. 2016). Additional remains are the female reproductive organs Weltrichia sp., scales of Cycadolepis type, and Cycadeospermum lovisatoi KRASSER, 1912 seeds 
(Tab 1). Otozamites veronensis DE ZIGNO, 1881 (Edwards 1929, Comaschia Caria 1959), originally described by Krasser (1913) as Otozamites lovisatoi KRASSER, 1913 is missing from the samples in the Prague collection. The illustration does, however, confirm its (former) existence.

Krasser (1920) indicated several additional taxa not confirmed during the revision of the various collections. These include Nilssonia compta (PHILliPs) BRONGNIART, 1828 (Krasser 1920: 11), Nageiopsis anglica SEWARD, 1900, Otozamites beanii (LindLey et HutTON) Brongniart, 1848, Ptilophyllum pecten (PHILlIPS) MORRIs, 1841 (Krasser 1920: 12), Taeniopteris vittata BRONGNIART, 1831 and Zamites sp. (Krasser 1920: 13). Also not confirmed are the reproductive organs Williamsonia leckenbyi NATHORST, 1911 (Krasser 1912, 1913), Williamsonia sewardii KRASSER, 1913, Williamsonia acuminata (DE ZIGNO) KRASSER, 1912 and the seed Cycadeospermum persicum KRASSER, 1912 (Tab. 1).

The conifers are among the most abundant groups in this plant assemblage. They are represented by shoots of Geinitzia divaricata (BUNBURY) HARRIS, 1979, Elatides williamsonii (LindLEY et HutTON) NATHORST, 1897, Elatocladus sp., Brachyphyllum expansum (Presl in STERnBerg) SEWARD, 1919 emend. Kendall 1949, Brachyphyllum sp. and Pagiophyllum sp. The wood fragment (Lov. B 128) might belong to Agathoxylon Hartig, 1848, the ovuliferous scales to Araucarites sardinicus (KRASSER) KRASSER, 1920. Brachyphyllum mamillare BRONGNIART, 1828 and Pityophyllum nordenskioldii (HEER) SEWARD, 1919, listed by Krasser (1913, 1920), cannot be confirmed for this flora.

Several seed types with unknown botanical affinity were also recovered from the plant assemblage, such as the seeds Carpolithes sp. 1 sensu Scanu et al. (2015) and Carpolithes sp. 2 sensu Scanu et al. (2015); Edwards (1929) suggested they might belong to Williamsonia. Elongate, entiremargined leaf fragments, with secondary veins perpendicular to the midvein, here attributed to Taeniopteris sp. (Tab. 1), are also of ambiguous botanical affinity, but probably belonging to the cycadophytes, whereas the stem fragments named Sardoa robitschekii KRASSER, 1920 are probably Bennettitales.

Here we do not consider the typical Triassic taxa taken by Tornquist (1904b), from a list of plant fossils identified by Sterzel, since those attributions were based on an erroneous stratigraphic allocation of the levels yielding plant fossils. This includes the sphenophyte stems Equisetum arenaceum (JAEGER) SCHENK, 1864, Equisetum mougeotii Brongniart, 1828 and Calamites sp., the fern stem Caulopteris sp., the seed fern leaf Sagenopteris rhoifolia PRESL, 1838 var. elongata BRAUN in MÜNSTER, 1843, the cycadophytes Otozamites beanii (LindLey et HutTon) Brongniart, 1849 and Pterophyllum sp. cf. P. braunianum GoEPPERT, 1844, and the conifers Voltzia sp. and Yuccites vogesiacus SCHIMPER et Mougeot, 1844. Also not considered is the work of Comaschi Caria (1959), which represents a compilation of all historical taxa names through time.

\section{Discussion of the three collections of plant fossils from Sardinia}

A recent revision (Scanu et al. 2015) of the Lovisato collection stored in Cagliari showed that the plant assemblage was composed of ferns (Phlebopteris polypodioides,
Hausmannia sp., Coniopteris sp. cf. C. hymenophylloides, Todites sp. cf. T. williamsonii, Cladophlebis sp.), seed ferns (Sagenopteris phillipsii, Ptilozamites sp. cf. P. blasii), cycadophytes (Nilssonia sp., Cycadeospermum lovisatoi, Pterophyllum sp. cf. P. thomasii, Ptilophyllum pectinoides, Ptilophyllum cariae, Williamsonia hildae, Weltrichia sp., Taeniopteris sp.), Czekanowskiales (Czekanowskia sp. cf. C. furcula), conifers (Geinitzia divaricata, Brachyphyllum expansum, Brachyphyllum sp., Elatocladus sp.) and several types of seeds (Carpolithes sp. 1, Carpolithes sp. 2). The revision of the Domenico Miccolis Collection (Scanu et al. 2016), stored at the Natural History Museum of Venice added several taxa to this list, including the ferns Dicksonia kendallii, Eboracia sp. cf. E. lobifolia, Marattia intermedia, Phlebopteris braunii and P. muensteri and the bennettitalean microsporangiate reproductive organ Weltrichia sp. cf. $W$. whitbiensis. This paper, dealing with the second part of the Lovisato collection deposited in the Faculty of Sciences, Charles University, Prague, adds to this list horsetails (Equisetites beanii, Equisetites columnaris), ferns (Cladophlebis denticulata, Hausmannia crenata, Phlebopteris dunkeri), ginkgophytes (Eretmophyllum lovisatoi), cycadophytes (Nilssonia sp. cf. N. orientalis, Cycadolepis sp., Ptilophyllum sp. cf. P. hirsutum), conifers (Araucarites sardinicus, Agathoxylon sp., Pagiophyllum sp.) and a putative bennettitalean stem (Sardoa robitschekii).

The floral composition of the various plant assemblages, considered in terms of the number of fossil taxa (Tab. 1) and not relative abundances is noticeably different. The Lovisato collection in Cagliari (Text-fig. 1a) is the richest in its number of fossil taxa (24). Cycadophytes (29.2\%) and ferns (25\%) are the two most abundant groups in the Lovisato collection, while conifers are common (16.7\%), horsetails and Czekanowskiales are rare (1 fossil taxon each: $4.2 \%)$ and ginkgophytes are absent. The Krasser collection (Lovisato B collection; Text-fig. 1b) in Prague is almost as diverse, with 23 fossil taxa (Text-fig. 1b). The three most abundant groups are the same as in the collection of Cagliari, although the conifers are the most diverse plant group (30.4\%), followed by cycadophytes and ferns (each represented by four taxa $(17.4 \%))$. Horsetails are represented by three fossil taxa and thus common, whereas Czekanowskiales are absent from the Lovisato B collection.

The Miccolis collection (Text-fig. 1c) is the least diverse plant assemblage, with only 11 fossil taxa. The most abundant are the ferns (63.6\%), while seed ferns, ginkgophytes and Czekanowskiales are absent. This difference in composition, especially of the Miccolis collection, could be the result of a collecting bias. The specimens of the Miccolis collection are mostly well preserved and beautiful, typical for a fossil collector (Scanu et al. 2016). The two plant assemblages collected by Lovisato apparently come from different stratigraphic levels, and perhaps different geographic locations. Moreover, the Lovisato B collection (Krasser collection in Prague) was sent as an addition to the previously studied Lovisato A collection, and is thus composed mostly of material Lovisato apparently considered different from the A collection. This might explain the contrasting compositions of the three plant assemblages. The total flora (Text-figs 1d, 2 ), based on the three collections together, is composed mostly of ferns $(32.6 \%)$, followed by cycadophytes $(26.1 \%)$ and conifers $(15.2 \%)$. 
Lovisato Cagliari coll. (Scanu et al. 2015)

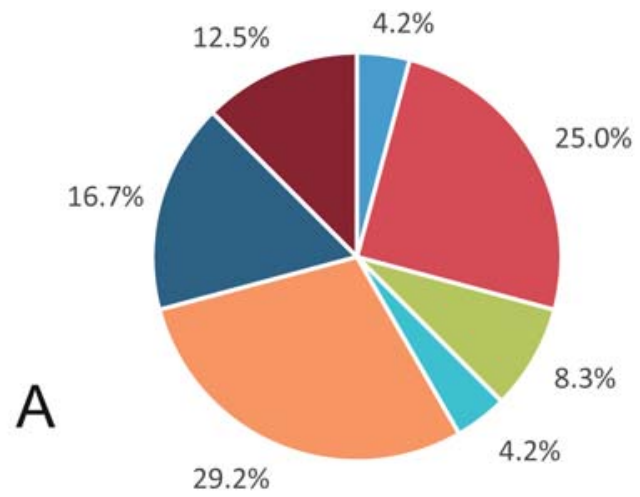

Miccolis Venice coll. (Scanu et al. 2016)

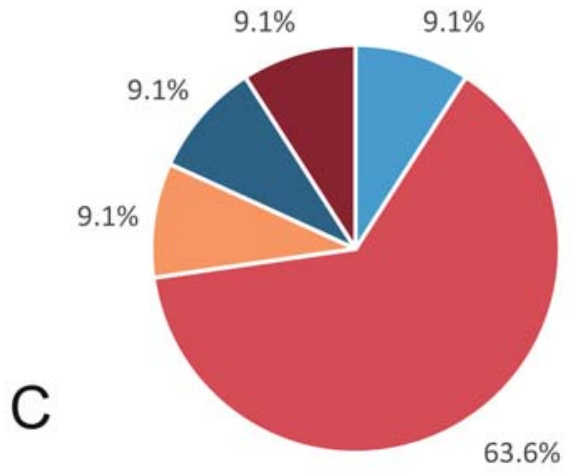

$63.6 \%$

\section{Krasser Prague (this work)}

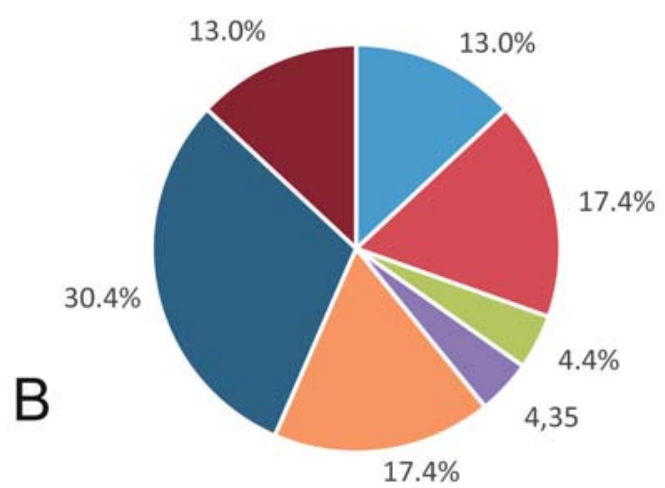

total (this work)

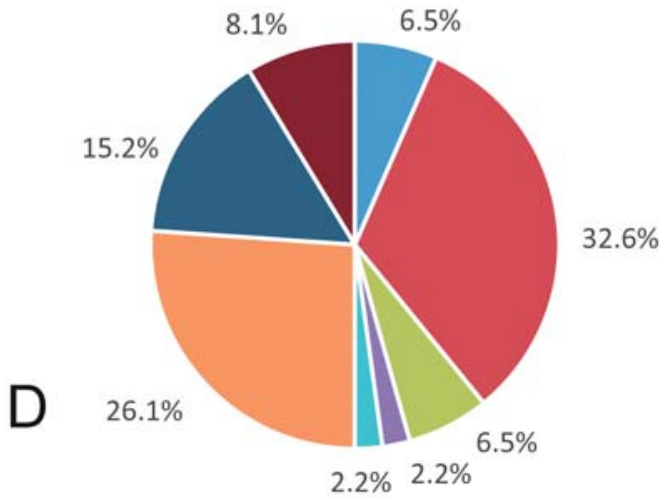

$$
\begin{aligned}
& \text { - Sphenophytes } \square \text { Pteridophytes } \square \text { Seed ferns } \square \text { Ginkgophytes }
\end{aligned}
$$

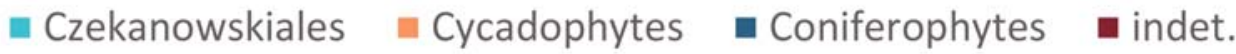

Text-fig. 1. Composition in taxa per plant group for different collections: a) composition of Lovisato A collection in Cagliari; b) composition of Lovisato B (Krasser) collection in Prague; c) composition of Miccolis collection in Venice; d) composition of total plant assemblage.

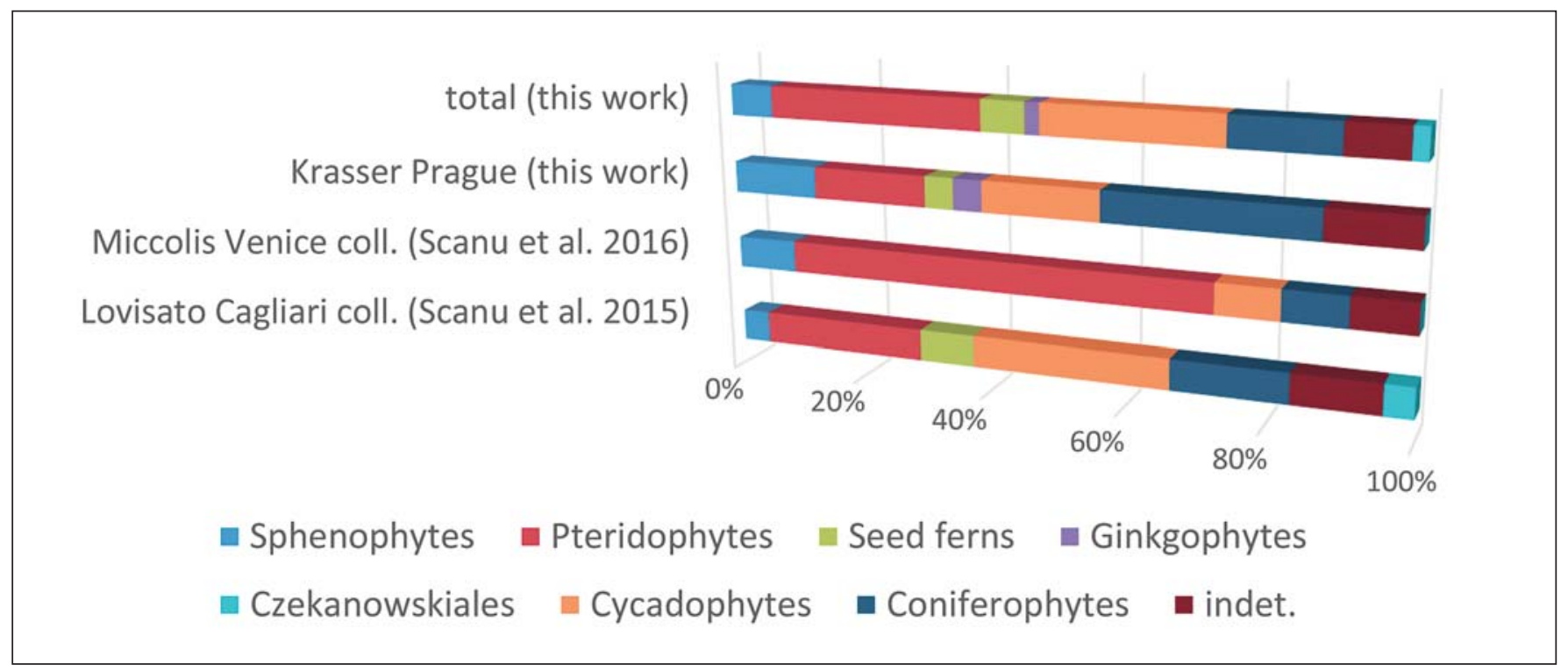

Text-fig. 2. Diagram showing contrast between compositions of the various collections and that of the total flora. 


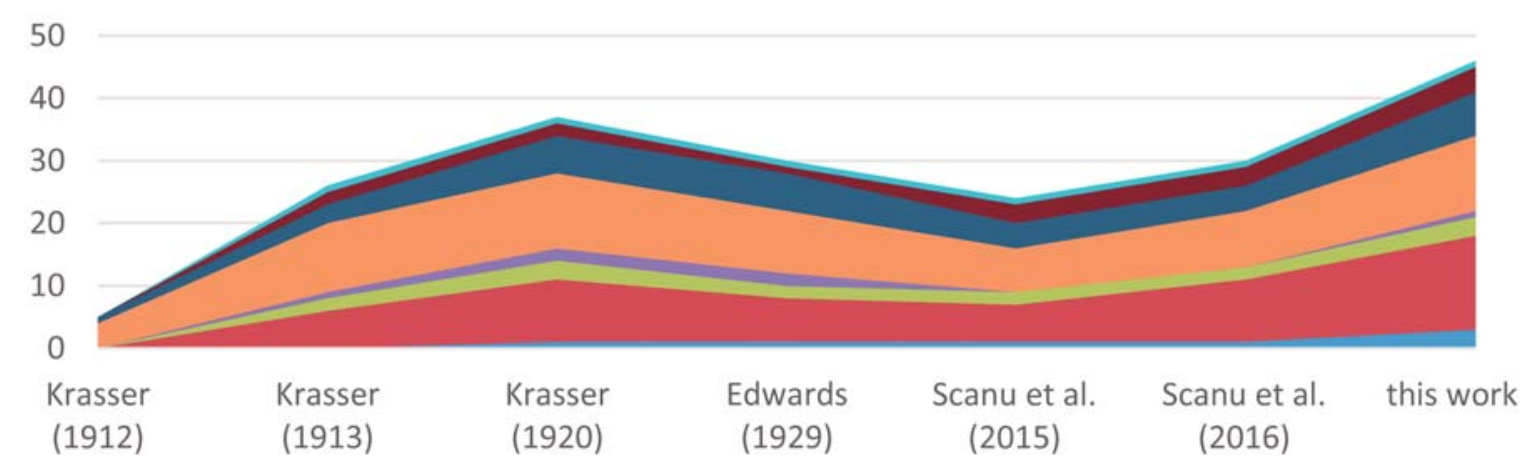

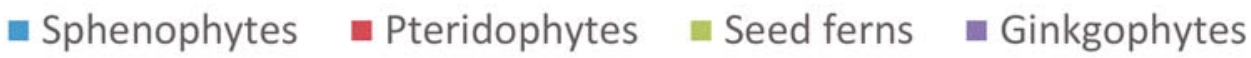

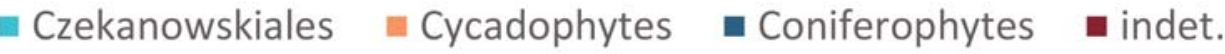

Text-fig. 3. Diagram showing how the composition of the Middle Jurassic flora of Sardinia changed through time, from papers of Krasser to more recent revisions (Krasser 1912, 1913, 1920, Edwards 1929, Scanu et al. 2015, 2016, this paper).

The known biodiversity of the Middle Jurassic flora of Sardinia has increased from 24 fossil taxa (Scanu et al. 2015) to 46 fossil taxa (this paper) just by adding further historical collections to our dataset (the original outcrops are no longer accessible, so no new taxa can be added by further sampling). By considering the papers starting with modern revisions, Text-fig. 3 shows how, from the few taxa described by Krasser (1912), there was a strong increase (from five fossil taxa to 37 fossil taxa) due to Krasser $(1913,1920)$ having studied more material. Edwards (1929) reduced the number of taxa with his revision of the collection stored in Prague. The study of the three collections carried out in the last few years (Scanu et al. 2015, 2016, this paper) has increased diversity again, up to 46 taxa in this paper. However, the curve still shows a definitive increase through time, indicating that the real biodiversity of the Middle Jurassic flora of Sardinia was somewhat higher (Text-fig. 3).

\section{Conclusions}

The Lovisato "collection B", described by Krasser (1920) and Edwards (1929), and preserved in the Faculty of Sciences, Charles University, Prague, differs noticeable from "collection A" described previously by Krasser (1912, 1913), and stored in the Cagliari University. Cycadolepis sp., Nilssonia sp. and Nilssonia sp. cf. N. orientalis, Pagiophyllum sp. and Agathoxylon sp. were previously not known from the Jurassic of Sardinia. On the other hand, Coniopteris sp. cf. C. arguta, Dictyophyllum rugosum, Klukia exilis, Laccopteris elegans and Laccopteris spectabilis (ferns), Sagenopteris goeppertiana and Gristhorpia nathorstii (seed ferns), Baiera phillipsii (ginkgophytes), Cycadeospermum persicum, Nageiopsis (= Otozamites) anglica, Nilssonia compta, Otozamites beanii, Otozamites veronensis, Ptilophyllum pecten, Taeniopteris vittata, Zamites sp., Williamsonia acuminata, Williamsonia leckenbyi and Williamsonia sewardii (cycadophytes), Brachyphyllum mamillare and
Pityophyllum nordenskioldii (conifers) could not be confirmed, because they were either missing from the collection or too badly preserved to confirm the determinations. Therefore, the Middle Jurassic flora of Sardinia currently comprises 46 fossil taxa. The three plant fossil collections differ noticeably. Cycadophytes (especially bennettitaleans) dominate the Cagliari collection, ferns the Venice collection, and conifers are the most important group (in diversity and abundance) in the Prague collection. Horsetails are the most abundant plant group in the Krasser collection, although not very diverse. A distinct collecting bias is postulated for the Venice collection, whereas the Cagliari and Prague collections might belong to different stratigraphic levels. Study of the three plant fossil collections indicates a diverse Middle Jurassic flora of Sardinia, although the hidden biodiversity is still very high. This underlines a general problem with allochthonous Palaeozoic and Mesozoic plant assemblages, that they provide a highly fragmented and incomplete picture of the past vegetation.

\section{Acknowledgements}

We thank Stephen McLoughlin (Stockholm) very much for his constructive reviews and linguistic corrections, as well as Mihai Popa and an anonymous reviewer for their constructive remarks. The Krasser material of Prague was studied by Evelyn Kustatscher during a visit to the National Museum in Prague under the project "The diversity of the Jurassic floras of Europe" (CZ-TAF-4535), which received funding through SYNTHESYS, which was made available by the European Community-Research Infrastructure Action under the FP7 "Structuring the European Research Area" Programme. GGS gratefully acknowledges the Sardinia Regional Government for its PhD scholarship (P.O.R. Sardegna F.S.E. Operational Programme of the Autonomous Region of Sardinia, European Social Fund 2007 - 2013 Axis IV Human Resources, Objective 1.3, Line of 
Activity 1.3.1). This paper is also part of the UNESCO-IUGS IGCP Project 632 "Continental Crises of the Jurassic: Major Extinction Events and Environmental Changes within Lacustrine Ecosystems". This work was financially supported by Ministry of Culture of the Czech Republic (DKRVO 2016/05, National Museum, 00023272).

\section{References}

Amadesi, E., Cantelli, C., Carloni, G. C., Rabbi, E. (1960): Ricerche geologiche sui terreni sedimentari del foglio 208 Dorgali [Geological research on the sedimentary outcrops of the sheet 208 Dorgali]. - Giornale di Geologia, 28: 59-87. (in Italian)

Barbacka, M., Bodor, E., Jarzynka, A., Kustatscher, E., Pacyna, G., Popa, M. E., Scanu, G. G., Thévenard, F., Ziaja, J. (2014): European Jurassic floras: Statistics and palaeoenvironmental proxies. - Acta Palaeobotanica, 54: 173-195. https://doi.org/10.2478/acpa-2014-0011

Bartiromo, A., Barone Lumaga, M. R. (2009): Taxonomical revision of the Collection of Jurassic plants from Roverè di Velo (Veneto, northern Italy) stored in the Palaeontological Museum of the University of Naples "Federico II". - Bollettino della Società Paleontologica Italiana, 48: $1-13$.

Batten, D. J. (1999): Small palynomorphs. - In: Jone, T. P. Rowe, N. P. (eds), Fossil plants and spores: Modern Techniques. Geological Society of London, London, pp. 15-19.

Bonci, M. C., Vannucci, G. (1986): I vegetali Sinemuriani di Osteno (Lombardia) [The Sinemurian plant fossils from Osteno (Lombardy)]. - Atti Società Italiana Scienze Naturali Museo Civico Storia Naturale, 127: 107-127. (in Italian)

Bravi, S. (1995): Studies on old and new Meso-Cenozoic "Plattenkalk" in Southern Italy. - In: Extended Abstracts, II International Symposium on Lithographic Limestones, $9^{\text {th }}-16^{\text {th }}$ July 1995 , Lleida - Cuenca, Spain, pp. 39-43.

Bravi, S., Garassino, A., Bartiromo, A., Audo, D., Charbonnier, S., Schweigert, G., Thévenard, F., Longobardi, C. (2014): Middle Jurassic Monte Fallano Plattenkalk (Campania, southern Italy): First report on terrestrial plants, decapod crustaceans and fishes. - Neues Jahrbuch für Geologie und Paläontologie, Abhandlungen, 272: 79-107. https://doi.org/10.1127/0077-7749/2014/0398

Cleal, C. J., Thomas, B. A., Batten, D. J., Collinson, M. E. (2001): Mesozoic and Tertiary Palaeobotany of Great Britain (Geological Conservation Review Series 22). Joint Nature Conservation Committee, Peterborough, xviii $+335 \mathrm{pp}$

Cocozza, T., Praturlon, A. (1966): Note geologiche sul colle Cantocchio (Lepini sud-occidentali, Lazio) [Geological notes on Colle Cantocchio (southwestern Lepini mountains, Lazio)]. - Geologica Romana, 5: 323-334. (in Italian)

Comaschi Caria, I. (1959): Le piante fossili della Sardegna [The fossil plants of Sardinia]. - Rivista Italiana di Paleontologia e Stratigrafia, Memorie, 7: 1-176. (in Italian)
Costamagna, L. G. (2015): Middle Jurassic continental to marine transition in an extensional tectonics context: the Genna Selole Fm depositional system in the Tacchi area (central Sardinia, Italy). - Geological Journal, 51: $722-736$. https://doi.org/10.1002/gj.2680

Costamagna, L. G., Barca, S. (2004): Stratigrafia, analisi di facies, paleogeografia ed inquadramento regionale della successione giurassica dell'area dei Tacchi (Sardegna Orientale) [Stratigraphy, facies analyses, palaeogeography and regional setting of the Jurassic successions of the Tacchi area (Eastern Sardinia)]. - Bollettino della Società Geologica Italiana, 123: 477-495. (in Italian)

de Zigno, A. (1856-68): Flora Fossilis Formationis Oolithicae. Le plantae fossili dell'Oolite. Vol. 1. - Tipografia del seminario, Padova, 225 pp. (in Italian)

de Zigno, A. (1873-85): Flora Fossilis Formationis Oolithicae. Le plantae fossili dell'Oolite. Vol. 2. Tipografia del seminario, Padova, 203 pp. (in Italian)

Del Rio, M. (1976): Analisi palinologica del Giurese della Sardegna centrale [Palynological analyses of the Jurassic of central Sardinia]. - Bollettino della Società Geologica Italiana, 95: 619-631. (in Italian)

Del Rio, M. (1984): Palynology of Middle Jurassic black organic shales of "Tacco di Laconi", Central Sardinia, Italy. - Bollettino della Società Paleontologica Italiana, 23: 325-342.

Dieni, I., Massari, F. (1985): Mesozoic of Eastern Sardinia. - In: Cherchi, A. (ed.), Guide Book, 19 ${ }^{\text {th }}$ European Micropaleontological Colloquium, 1-10 October 1985. AGIP, Cagliari, pp. 66-78.

Dieni, I., Fisher, J. C., Massari, F., Salard-Cheboldaeff, M., Vozenin-Serra, C. (1983): La succession de Genna Selole (Baunei) dans le cadre de la paléogéographie mésojurassique de la Sardaigne orientale. - Memorie di Scienze Geologiche, Padova, 36: 117-148.

Edwards, W. N. (1929): The Jurassic flora of Sardinia. Annals and Magazines of Natural History, 10: 385-394. https://doi.org/10.1080/00222932908673071

Grandori, L. (1913a): Intorno alla flora dei calcari grigi studiata da Achille De Zigno. Nota preliminare [The fossil flora of the Calcari Grigi studied by Achille de Zigno. Preliminary note]. - Atti della Accademia scientifica veneto-trentino-istriana, Padova, 6: 143-148. (in Italian)

Grandori, L. (1913b): La Flora dei Calcari Grigi del Veneto, Parte Prima. Revisione e completamento dell'opera "Flora Fossilis Formationis Ooliticae" del Barone A. De Zigno [The flora oft he Calracri Grigi in Veneto. First part. Revision and conclusion of the work "Flora Fossilis Ooliticae" of Baron A. de Zigno]. - Memorie dell'Istituto Geologico della Regia Università di Padova, 2: 1-112. (in Italian)

Gothan, W. (1914): Die unter-liassische (rhätische) Flora der Umgegend von Nürnberg. - Abhandlungen der Naturhistorischen Gesellschaft zu Nürnberg, 19: 91-186.

Harris, T. M. (1961): The Yorkshire Jurassic Flora I. Tallophyta-Pteridophyta. - British Museum (Natural History), London, 212 pp. https://doi.org/10.5962/bhl.title.118957 
Harris, T. M. (1964): The Yorkshire Jurassic Flora II. Caytoniales, Cycadales and Pteridosperms. - British Museum (Natural History), London, 191 pp.

Harris, T. M. (1969): The Yorkshire Jurassic Flora III. Bennettitales. - British Museum (Natural History), London, $186 \mathrm{pp}$.

Harris, T. M. (1979): The Yorkshire Jurassic Flora V. Coniferales. - British Museum (Natural History), London, $167 \mathrm{pp}$.

Harris, T. M. Millington, W., Miller, J. (1974): The Yorkshire Jurassic Flora IV. 1. Ginkgoales, 2. Czekanowskiales. British Museum (Natural History), London, 150 pp.

Jarzynka, A., Pacyna, G. (2015): Fossil flora of Middle Jurassic Grojec clays (southern Poland). Raciborski's original material reinvestigated and supplemented. I. Sphenophytes. - Acta Palaeobotanica, 55(2): 149-181. https://doi.org/10.1515/acpa-2015-0013

Kendall, M. W. (1949): On Brachyphyllum expansum (Sternberg) Seward, and its cone. - Annals and Magazine of Natural History, Series 12, 2: 308-320.

Kerp, H. (1990): The study of fossil gymnosperms by means of cuticular analysis. - Palaios, 5: 548-569.

https://doi.org/10.2307/3514861

Krasser, F. (1912): Williamsonia in Sardinien. - Sitzungsberichte der kaiserlichen Akademie der Wissenschaften, Mathematisch-naturwissenschaftliche Klasse, 121: 943973.

Krasser, F. (1913): Die fossile Flora der Williamsonien bergenden Juraschichten von Sardinien. - Anzeiger der kaiserlichen Akademie der Wissenschaften, Mathematisch-naturwissenschaftliche Klasse, 50(4): 31-36.

Krasser, F. (1920): Doggerflora von Sardinien. - Sitzungsberichte, Akademie der Wissenschaften in Wien, Mathematisch-naturwissenschaftliche Klasse, 129: 3-26.

Meneghini, G. (1857) : Paléontologie de l'île de Sardaigne ou description des fossiles recueillis dans cette contrée par le général Albert Lamarmora. - In: Lamarmora, A. (ed.), Voyage en Sardaigne, partie 3, tome II. Imprimerie Royal, Turin, pp. 81-650.

Popa M. E., McElwain, J. C. (2009): Bipinnate Ptilozamites nilssonii from Jameson Land and new considerations on the genera Ptilozamites Nathorst 1878 and Ctenozamites Nathorst 1886. - Review of Palaeobotany and Palynology, 153: 386-393.

https://doi.org/10.1016/j.revpalbo.2008.10.007

Popa, M. E., Meller, B. (2009): Review of Jurassic plants from the Anina (Steierdorf) Coal Mining Area, South Carpathians, in the collections of the Geological Survey of Austria. - Jahrbuch der Geologischen Bundesanstalt, 149(4): 487-498.

Pott, C., McLoughlin, S. (2011): The Rhaetian flora of Rögla, northern Scania, Sweden. - Palaeontology, 54: 1025-1051. https://doi.org/10.1111/j.1475-4983.2011.01090.x

Praturlon, A. (1965): Resti di conifere al passaggio GiureseCretaceo nei Monti Lepini [Coniferal remains at the Jurassic-Cretaceous transition in the Lepini mountains]. - Bollettino della Società Geologica Italiana, 84: 279-287. (in Italian)

Praturlon, A. (1968): Cycadophyta and Coniferophyta from the Lias of M. Palombo (Marsica, Central Appennines). - Geologica Romana, 7: 1-26.
Salard-Cheboldaeff, M., Vozenin-Serra, C. (1984): Palynoflore et taphoflore Jurassique de Sardaigne CentreOrientale. - Revue de Paléobiologie, volume spécial, 193-197.

Scanu, G. G., Kustatscher, E., Pittau, P. (2012): The Jurassic plant fossils of the Lovisato Collection: preliminary notes. - Bollettino della Società Paleontologica Italiana, 51: 71-84.

Scanu, G. G., Kustatscher, E., Pittau, P. (2015): The Jurassic Flora of Sardinia - a new piece in the palaeobiogeography puzzle of the Middle Jurassic. - Review of Palaeobotany and Palynology, 218: 80-105. https://doi.org/10.1016/j.revpalbo.2014.08.008

Scanu, G. G., Kustatscher, E., Pittau, P., Van Konijnenburg-van Cittert J. H. A. (2016): New insight into the Middle Jurassic Floras of Sardinia (Italy) - The Miccolis Collection at the Museo di Storia Naturale of Venice, Italy. - Bollettino della Società Paleontologica Italiana, 55: 29-45.

Schweitzer, H.-J., Kirchner, M., Van Konijnenburg-van Cittert, J. H. A. (2000): The Rhaeto-Jurassic flora of Iran and Afghanistan. 12. Cycadophyta II. Nilssoniales. Palaeontographica, Abteilung B, 254(1-3): 1-63.

Schweitzer, H.-J., Schweitzer, U., Kirchner, M., Van Konijnenburg-van Cittert, J. H. A., Van der Burgh, J., Ashraf, R. A. (2009): The Rhaeto-Jurassic flora of Iran and Afghanistan. 14. Pterophyta - Leptosporangiatae. Palaeontographica, Abteilung B, 279: 1-108.

Schweitzer, H.-J., Van Konijnenburg-van Cittert, J. H. A., van der Burgh, J. (1997): The Rhaeto-Jurassic flora of Iran and Afghanistan. 10. Bryophyta, Lycophyta, Sphenophyta, Pterophyta-Eusporangiatae and Protoleptosporangiatae. - Palaeontographica, Abteilung B, 243: 103-192.

Sordelli, F. (1896): Studi sulla vegetazione di Lombardia durante i tempi geologici [Studies on the vegetation in Lombardy during geologic times]. - L. F. Cogliati, Milano, 298 pp. (in Italian) https://doi.org/10.5962/bhl.title.109282

Tornquist, A. (1902): Ergebnisse einer Bereisung der Insel Sardiniens. - Sitzungsberichte der Königlich Preussischen Akademie der Wissenschaften zu Berlin, 35: 808-829.

Tornquist, A. (1904a): Die Gliederung und Fossilführung der ausseralpinen Trias auf Sardinien. - Sitzungsberichte der Königlich Preussischen Akademie der Wissenschaften zu Berlin, 38: 1098-1117.

Tornquist, A. (1904b): Beiträge zur Geologie der Westlichen Mittelmeerland. I: Die Pflanzen des Mitteljurassischen Sandsteines Ostsardinien. - Neues Jahrbuch für Mineralogie, Geologie und Paläontologie, 20: 149-158.

Van Konijnenburg-van Cittert, J. H. A., Morgans, H. S. (1999): The Jurassic Flora of Yorkshire (Field Guide to Fossil, No. 8). - The Palaeontological Association, London, $134 \mathrm{pp}$.

Van Konijnenburg-van Cittert, J. H. A. (2008): The Jurassic fossil plant record of the UK area. - Proceedings of the Geologists'Association, 119: 59-72. https://doi.org/10.1016/S0016-7878(08)80258-1

Weber, R. (1968): Die fossile Flora der Rhät-Lias Übergangsschichten von Bayreuth (Oberfranken) unter besonderer 
Berücksichtigung der Coenologie. - Erlanger Geologische Abhandlungen, 72: 1-73.

Wesley, A. (1956): Contribution to the knowledge of the flora of the Grey Limestone of Veneto, I. - Memorie dell' Università Padova, 19: 1-69.

Wesley, A. (1958): Contribution to the knowledge of the flora of the Grey Limestone of Veneto, II. - Memorie dell'Università Padova, 21: 1-57.

Wesley, A. (1966): The fossil flora of the Grey Limestones of Veneto, Northern Italy, and its relationships to the other European floras of similar age. - The Palaeobotanist, 14: 124-130.

Wesley, A. (1974): On the bennettitalean remains from the Lias of northern Italy. - In: Bose, M. N. (ed.), Symposium on morphological and stratigraphical palaeobotany. Birbal Sahni Institute of Palaeobotany, Lucknow, Special Publication no. 2, pp. 66-71.

\section{Explanations to the plates}

\section{PLATE 1}

Sphenophytes and ferns

1. Equisetites columnaris (BRONGNIART) PHILliPs; stem fragment with two leaf sheaths; Lov. B 2.

2. Equisetites columnaris (BRONGNIART) PhILliPs; nodal fragment with leaf traces; Lov. B 9.

3. Equisetites columnaris (BRONGniart) PhILlips; stem fragment; Lov. B 10

4. Equisetites beanii (BUNBURY) SEWARD; stem fragment; Lov. B 17.

5. Equisetites beanii (BUNBURY) SEWARD; stem fragment; Lov. B 17.

6. Phlebopteris dunkeri (SCHENK) SCHENK; frond fragment with partially preserved pinnae; Lov. B 64.

7. Phlebopteris dunkeri (SCHENK) SCHENK; pinna fragment showing reticulate venation; Lov. B 73.

8. Phlebopteris polypodioides BRONGNIART; fertile pinnae fragment indicating position of sori; Lov. B 95.

9. Todites williamsonii (BRONGNIART) SEWARD; small sterile fragment showing venation; Lov. B 16.4.

10. Sphenophyte nodal fragment with attached microphylls; possibly Schizoneura sp.; Lov. B 49.1.

11. Sphenophyte nodal fragment with attached microphylls; possibly Schizoneura sp.; Lov. B 49.2.

All scale bars $-10 \mathrm{~mm}$.

\section{PLATE 2}

Seed ferns, Cycadophyta, Ginkgophyta and incertae sedis

1. Nilssonia sp. cf. $N$. orientalis HEER; leaf fragment with undivided secondary veins; Lov. B 35.

2. Sagenopteris sp.; fragment of leaflet, with clear venation; Lov. B 35.

3. Sardoa robitschekii KRASSER; stem fragment; Lov. B 46.

4. Ptilophyllum sp.; leaf fragment with slightly falcate segments; Lov. B 65.

5. Eretmophyllum lovisatoi EDWARDs; partially preserved leaf used for cuticular identification; Lov. B 66.1.

6. Ptilophyllum sp.; leaf fragment; Lov. B 63.1.

7. Eretmophyllum lovisatoi EDWARDS; partially preserved leaf, Lov. B 67.

All scale bars $-10 \mathrm{~mm}$.

\section{PLATE 3}

Branchiopods and conifers

1. Geinitzia divaricata (BUNBURY) HARRIS; large shoot fragment with clear leaf scars; Lov. B 29.

2. Araucarites sardinicus (KRASSER) KRASSER; ovuliferous scale with single, large seed; Lov. B 56.

3. Araucarites sardinicus (KRASSER) KRASSER; ovuliferous scale with single, large seed; Lov. B 50.

4. Branchiopod, formerly called Estheria; Lov. B 98.

5. Elatides williamsonii (LindLey et HutTON) NATHORST; shoot fragment; Lov. B 72.

6. Branchiopod, formerly called Estheria; Lov. B 99.

7. Geinitzia divaricata (BUNBURY) HARRIS; shoot fragments with leaves; Lov. B 92.1.

8. Pagiophyllum sp., shoot fragment with very small leaves; Lov. B 99.

9. Elatides williamsonii (Lindley et Hutton) NATHORst; shoot fragment; Lov. B 122.

All scale bars $-10 \mathrm{~mm}$. 
PLATE 1
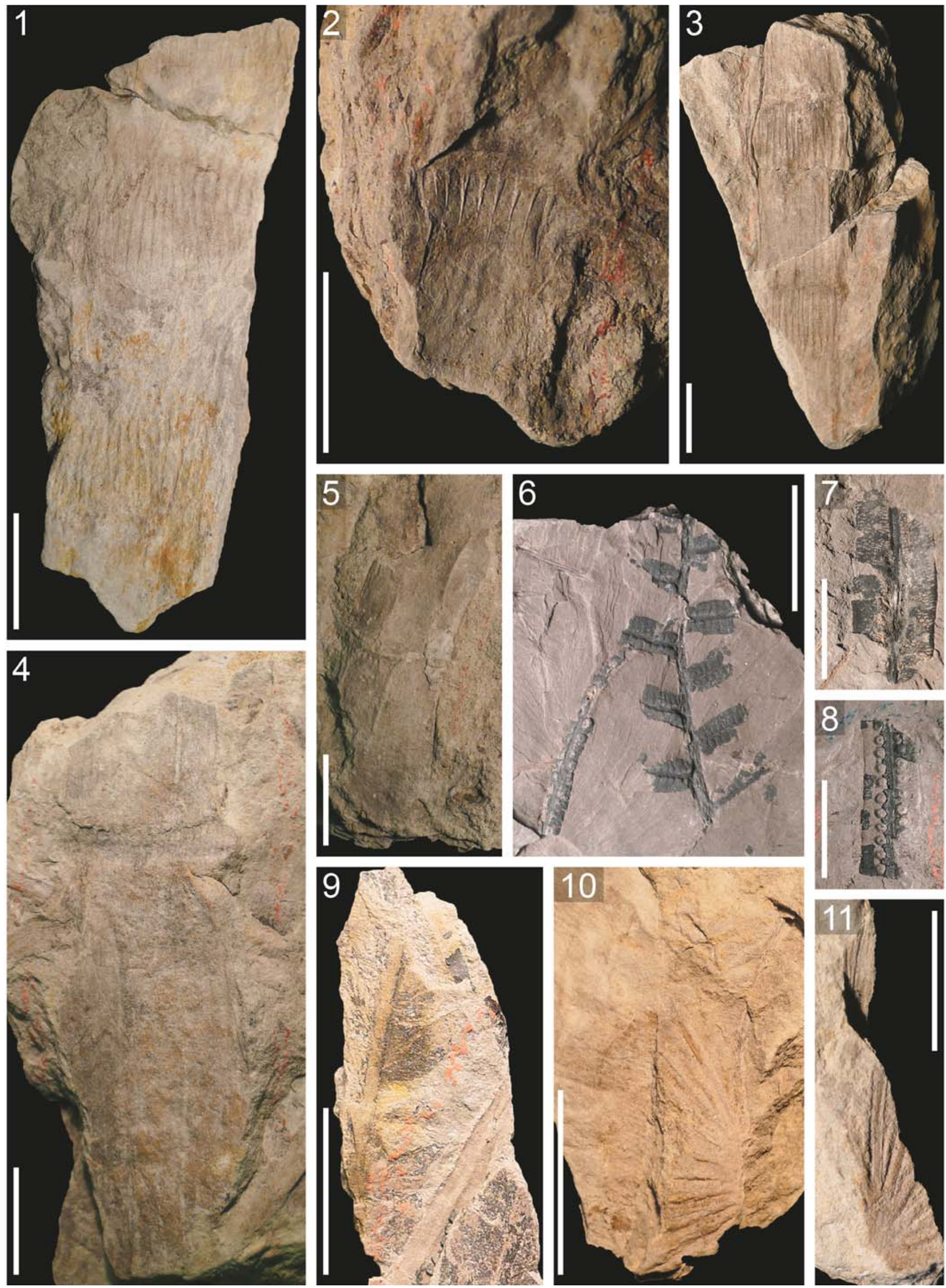
PLATE 2

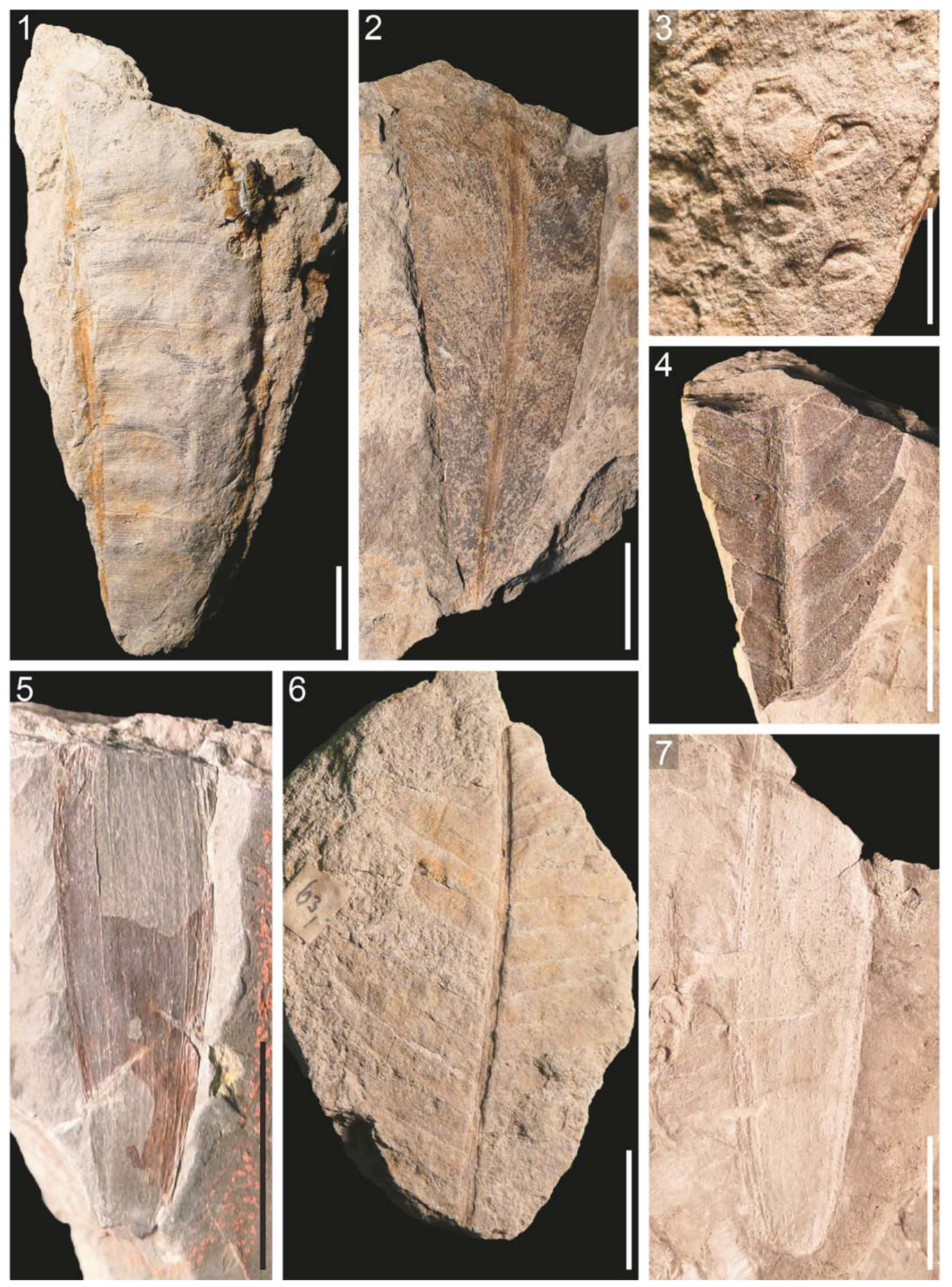




\section{PLATE 3}
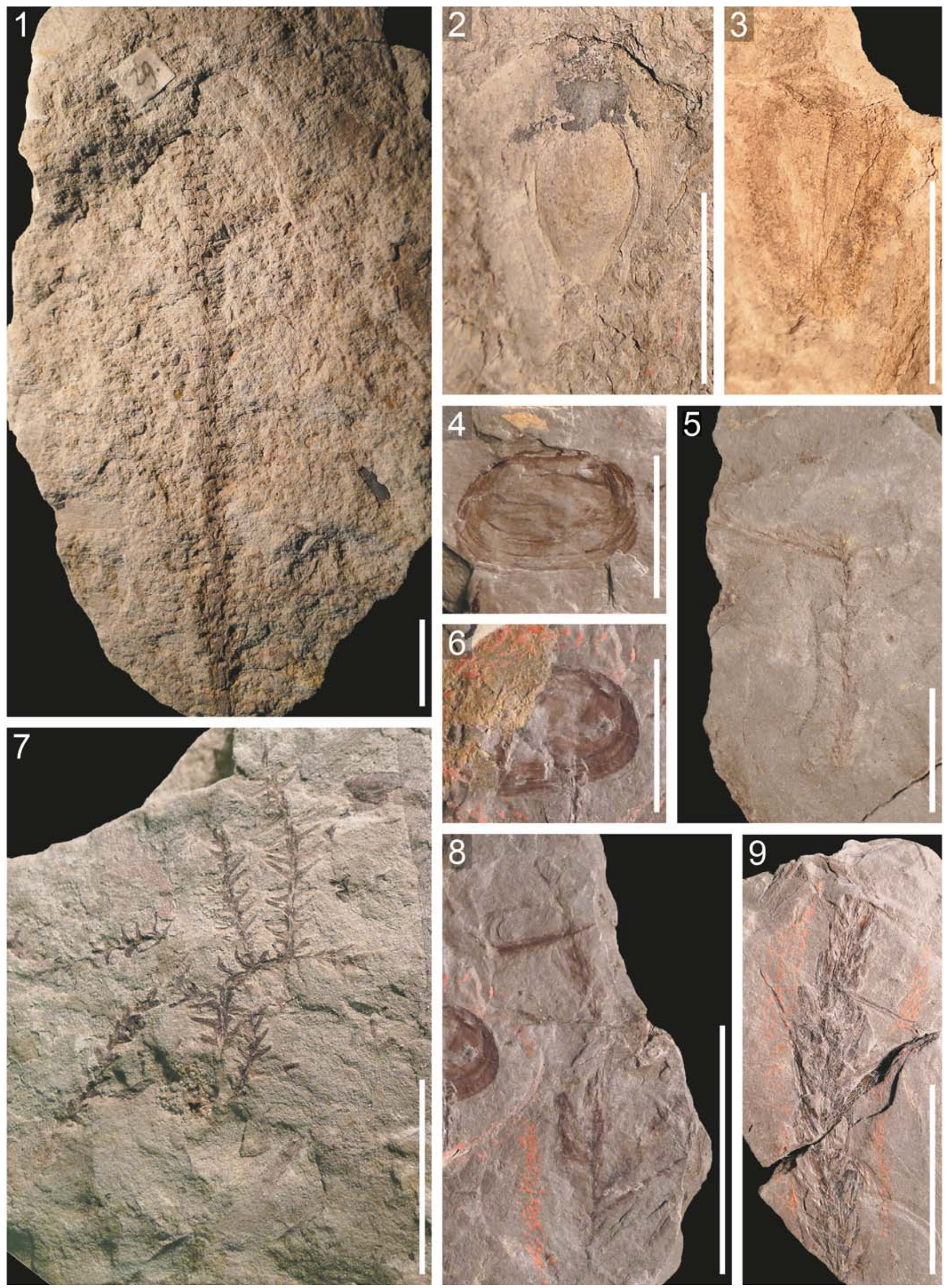